$466-3$

\title{
comprobación experimental de cubiertas laminares, por medio de modelos reducidos
}

\section{BENITO}

Ingeniero de Caminos Canales y Puertos

Encargado de la Sección de Modelos Reducidos de Estructuras

del Laboratorio Central

de Ensayo de Materiales de Construcción

"The problem of covering large areas using the minimun of intermediate supporting members is one which has appealed to architects and engineers for centuries..."

$$
\text { E. D. MILLS }
$$

\section{IV. modelos en rotura}

En los modelos de que se ha tratado en el capítulo anterior, el material era de características homogéneas en todo el espesor de la lámina. Aunque se ha mostrado su utilidad en la comprobación experimental de teorías que admitan la citada hipótesis de homogeneidad, su comportamiento resistente no tiene por qué ser idéntico al de la obra real, si ésta es de hormigón armado o pretensado. Parece, por tanto, interesante el estudio, por medio de modelos reducidos, de cubiertas laminares, construídas con un material anisótropo, compuesto por hormigón y armaduras de acero y en los que se pueda estudiar, no sólo su comportamiento resistente en período elástico, sino además observar la fisuración y el colapso de la estructura, para, de ello, deducir su forma de trabajar bajo la acción de las cargas y, sobre todo, cual es el coeficiente de seguridad real de la obra a la rotura.

En el presente capítulo se describe la construcción y ensayo de este tipo de modelos, así como algunos ejemplos realizados por nosotros en el Laboratorio Central de Madrid. 


\section{Construcción de los modelos}

Si se desea que el modelo represente una estructura de hormigón armado, al estar sometida a unas cargas previstas, y esto, tanto durante el período elástico, como cuando se produce la fisuración y la rotura por incremento de las cargas, es indispensable que la relación entre tensiones y deformaciones originadas en el modelo sea, en todo, análoga a la obtenida en la estructura real. Y si, además, se desea estudiar los fenómenos de pandeo, de importancia primordial en las estructuras laminares, y en los que influyen de modo importante los cambios de forma que adopta la estructura como consecuencia de las cargas, se deduce del Análisis Dimensional que los valores de los módulos de elasticidad longitudinal y de los coeficientes de Poisson de los materiales utilizados en el modelo y en la obra real deben ser iguales.

La necesidad de obtener un material que cumpliese con todas estas condiciones $\mathrm{y}$ en el que las rigideces relativas, en cuanto al espesor, se mantuviesen en la misma proporción, que en la obra real producen la unión del hormigón con las armaduras, nos llevó a estudiar la posibilidad de realizar los modelos reducidos, con un hormigón cuyo tamaño máximo de árido fuese muy pequeño, con el fin de permitirnos hormigonar los reducidos espesores que tendría el modelo. Siguiendo este razonamiento hemos utilizado, para los modelos, un mortero compuesto de cemento y arena, convenientemente dosificado para que las cargas de rotura a tracción y compresión fuesen iguales a las correspondientes al hormigón en obra para tiempos análogos. La constitución física de este material y una adecuada dosificación, nos aseguraban que los módulos de elasticidad y coeficientes de Poisson serían de igual valor en el modelo y en la obra real.

Dentro de este mortero se pueden colocar las armaduras de acero, reducidas a escala, que hubiesen sido previstas para la obra real. Es suficiente sustituir dichas armaduras, por alambre de acero de pequeño diámetro, de fácil adquisición en el mercado. Estos alambres de acero suelen tener un límite elástico y una carga de rotura mayores que los utilizados como armaduras en el hormigón armado. Sin embargo, últimamente hemos logrado superar esta dificultad sometiendo los citados alambres a un tratamiento térmico, mediante el cual se disminuyen los valores de aquellas características, que quedan sensiblemente iguales a las de las armaduras utilizadas en la obra real.

Así como estas armaduras pueden reproducirse con suficiente precisión, no suele ocurrir lo mismo con los cables previstos en las cubiertas de hormigón pretensado, pues el gran número de aparatos de enclaje, que muchas veces no pueden reducirse a escala, obliga a cambiar el número y distribución de los cables previstos en el proyecto de la estructura real. En estos casos, la variación necesaria (previa consulta con el autor del proyecto) suele consistir en reducir el número de cables, obligando a que coincidan las resultantes de los esfuerzos transmitidos por éstos a la lámina, en el modelo y en la realidad.

Elegido el material, la elección de la escala apropiada del modelo es más sencilla. Los espesores de las láminas, que son pequeños, se hacen 
todavía menores en el modelo, y suelen ser, frecuentemente, los que limitan la escala. No puede olvidarse que en ese espesor van las armaduras, algunas veces en dos capas o mallas, entre las que es necesario colocar los cables para el postensado de la cubierta.

Para fabricar el modelo se utiliza un encofrado, el cual suele ser de yeso recubierto con una capa de laca ligeramente engrasada para evitar que el cemento se adhiera a él. Sobre el encofrado van sujetos un gran número de clavos, cuyas puntas salen del mismo en una longitud igual al espesor de la lámina. Estos clavos cumplen con una doble finalidad: Sirven de referencia para asegurar que el espesor de la lámina es el deseado y, así, hemos llegado a construir modelos de diferentes espesores (el más delgado de 6 milímetros), sin que el error cometido corrientemente haya sido superior a unas décimas de milímetro (y siempre inferior a medio milímetro). Esto se ha comprobado en los modelos después de rotos, en los que es siempre posible medir los espesores de la lámina en diferentes partes. Pero, además, al desencofrar, los clavos que atraviesan la lámina dejan orificios, y por ellos pueden pasar los alambres de los que cuelgan los pesos utilizados para cargar la cubierta.

\section{Procedimiento de carga}

Construído el modelo, es necesario aplicarle las cargas con un reparto análogo al que ha de tener una obra; pero, al mismo tiempo, con un ritmo de aplicación también semejante al de la realidad.

Como ya se indicó al tratar el mismo tema en el capítulo anterior, el procedimiento más práctico para aplicar cargas repartidas en una cubierta, consiste en colgar un gran número de pesos de la misma. Este método, además de no dificultar la colocación de aparatos de medida sobre la superficie del modelo, como ocurre cuando se aplican las cargas directamente sobre ella, tiene ahora otra gran ventaja, porque permite observar las superficies de la cubierta cargada, detectar la aparición de fisuras y grietas, y seguir con todo cuidado el proceso de rotura.

Para colocar las cargas, en el Laboratorio Central, desde hace varios años-y siempre con buenos resultados-venimos utilizando el procedimiento siguiente:

Se construye el modelo que se deba ensayar sobre un depósito de $2 \mathrm{~m}$ de profundidad y planta algo superior a la del modelo; en su interior se sitúan recipientes cilíndricos de $9 \mathrm{~cm}$ de diámetro, $1,60 \mathrm{~m}$ de longitud y de $9 \mathrm{~kg}$ de peso, que, al llenar el depósito con agua, quedan flotando. Estos recipientes se cuelgan convenientemente de la estructura que se ha de en- 
sayar; después, al vaciar el depósito todo lo lentamente que se desee, la estructura se va cargando progresiva y uniformemente en toda su superficie.

Colgando estos recipientes, adecuadamente distanciados, pueden obtenerse diversas disposiciones del reparto de la carga total. Si se colocan muy próximos, puede llegarse hasta una carga de una tonelada por metro cuadrado, y descargar o cargar la cubierta tantas veces como se desee. Si se precisan sobrecargas mayores, basta lastrar los depósitos convenientemente. En los ensayos realizados en el Laboratorio Central, se ha llegado a cargas ligeramente superiores a los $1.700 \mathrm{~kg} / \mathrm{m}^{2}$ rellenando los recipientes con gravilla. Como es lógico, al llenar el depósito general de agua, los recipientes así lastrados no flotan y la lámina no se descarga por completo de peso, por lo que es conveniente recurrir a esta solución solamente cuando se desee llegar hasta la rotura de la lámina, y hacer variar la sobrecarga entre 0 y 1 ton $/ \mathrm{m}^{2}$ para los ensayos repetidos.

Este sencillo procedimiento requiere la única construcción auxiliar de un depósito de fábrica de ladrillo, cuyo coste es muy reducido, y un gran número de recipientes cilíndricos, que se amortizan fácilmente, puesto que son aprovechables en sucesivos ensayos.

\section{Aparatos de medida}

En un modelo reducido de una cubierta de hormigón armado o pretensado, pueden medirse fácilmente corrimientos o flechas con un error de $0,01 \mathrm{~mm}$ y giros con una precisión de 0,0001 radiantes, precisión que juzgamos suficiente en la práctica. La medida de deformaciones por medio de elongámetros mecánicos o eléctricos es también posible en las superficies de las láminas, aunque su utilidad es más discutible por tratarse de un material anisótropo.

Cuando la cubierta es pre o postensada, es fácil anclar este tipo de armaduras por medio de dinamómetros visibles durante el ensayo, y que, por tanto, permiten conocer las cargas que en ellas actúan.

Las magnitudes de los giros, corrimientos y deformaciones medidas, obtenidas con sobrecargas crecientes y decrecientes, dan una idea clara del comportamiento elástico de la estructura, y, además, sus valores numéricos son de indudable interés si se comparan con los que puedan obtenerse partiendo de las teorías más o menos simplificadas utilizadas por el proyectista. Todo esto permite formar un criterio sobre la validez de aplicación de dichas teorías al caso estudiado. Pero, además, si se aumentan lentamente las cargas hasta la aparición de fisuras y grietas, es posible, retirarlas después de ver 
cuáles son las grietas que se cierran y qué otras permanecen abiertas; interpretar estos fenómenos y, por último, cargar nuevamente la estructura hasta llegar a la rotura total, cuya forma suele ser muy aleccionadora.

Construídos estos modelos con un mortero de igual módulo de elasticidad y las mismas tensiones de rotura que las previstas para el hormigón de la obra real, las cargas repartidas por metro cuadrado en ésta deben ser iguales en el modelo; y en las referidas condiciones, la carga repartida capaz de producir la rotura del modelo será igual a la que causaría la ruina de la estructura real, con lo que resulta determinado experimentalmente su coeficiente de seguridad a rotura. Conviene advertir que en la carga de rotura estará incluída la compuesta por los pesos colgados, y el propio del modelo, que por unidad de superficie guarda la misma relación con el real que los espesores.

\section{Ensayos realizados}

A título de ejemplo se describen, brevemente, a continuación, tres estudios de otras tantas cubiertas laminares experimentadas por nosotros en modelo reducido.

Para cubrir el espacio central de la Iglesia de los Santos Félix y Régula, en Zürich, el Dipl. Ing. E. Schubiger, con el asesoramiento del Prof. Torroja, proyectó una cúpula de hormigón armado, de planta elíptica, postensada en su contorno, que iba colgada de una estructura cuya forma puede apreciarse en la figura 28.

El eje mayor de la elipse medía $24 \mathrm{~m}$; el menor, $17 \mathrm{~m}$; y la flecha en el centro, $1,6 \mathrm{~m}$. Por tanto, la relación flecha/diámetro mayor era de $1 / 15$, lo que expresa, claramente, la pequeña curvatura de la cúpula. En este tipo de estructuras, la máxima carga admisible viene limitada por iniciación de fenómenos de pandeo, que deben producirse en la dirección en que la curvatura sea menor. Dada la dificultad que presenta el cálculo riguroso de la carga crítica de pandeo, el autor del proyecto nos encargó la construcción y ensayo de tres cúpulas en modelo reducido. Los modelos se realizaron a escala $1 / 10$, y en ellos se reprodujeron todas las armaduras, así como los anillos de postensado, que fueron unidos a la lámina por dispositivos graduables, que permitieron variar los esfuerzos de postensado. En la figura 29 puede verse uno de los modelos durante un ensayo de carga.

Se midieron flechas y giros en la cúpula y deformaciones en los anillos, con cargas simétricas y disimétricas. Se dedujo el esfuerzo de postensado necesario para descimbrar la cúpula por elevación de la clave. Se estudiaron 
los efectos que podrían producirse en la realidad por un pequeño error en la nivelación de los apoyos, y, por último, se llegó en los tres modelos hasta la rotura, con una carga uniformemente repartida. La figura 30 representa la forma de rotura de uno de los modelos, y en ella se aprecia fácilmente la dirección en que se produjo el pandeo de la cubierta. La carga que originó el pandeo pudo ser determinada con toda precisión, gracias al sistema anteriormente descrito. La figura 31 es un detalle de la obra terminada $\left(^{*}\right)$.

En el segundo ejemplo, para cubrir las instalaciones de una gran fábrica holandesa, que ocupan en planta un rectángulo de $80 \times 264 \mathrm{~m}$, el Prof. Haas, de la Escuela Técnica Superior de Delft, ha proyectado una cubierta laminar de hormigón armado y postensado. Esta cubierta, cuya forma puede observarse en la figura 32 , está compuesta por 22 elementos iguales de $12 \times 80$ metros, y se apoya sobre tres pórticos múltiples.

La lámina de cada elemento trabaja como viga continua de dos vanos de $40 \mathrm{~m}$, con sólo $2 \mathrm{~m}$ de flecha, $7 \mathrm{~cm}$ de espesor, rigidizada con nervios cada 4 metros (**).

El cálculo del coeficiente de seguridad al pandeo, utilizado por el proyectista, se aplicaba, por primera vez, a una lámina de este tipo y dimensiones tan grandes. El autor de dicho trabajo quiso comprobar experimentalmente los resultados del cálculo; para ello, encargó al Laboratorio Central de Madrid la construcción y ensayo hasta rotura del modelo reducido de uno de los elementos estructurales.

El modelo (fig. 33) fué construído a escala $1 / 10$, reproduciendo su forma y dimensiones, tanto en su aspecto exterior como en las armaduras (se colocaron más de 7.500 armaduras de espesores reducidísimos). De acuerdo con el Prof. Haas, los cables necesarios para el postensado fueron sustituídos por alambres de acero de alta resistencia.

Durante los ensayos se aplicaron a la cubierta cargas crecientes y decrecientes, midiéndose corrimientos y giros en un gran número de puntos de la estructura. Se observó la aparición de fisuras, y, por último, se llegó hasta la rotura. La figura 34 representa al modelo después de roto, y la figura 35 es un aspecto de la estructura, en construcción, realizada por Nedam Havenwerke $\left({ }^{* * *}\right)$.

Como último de los ejemplos que exponemos aquí, en una nave de $48 \times 16 \mathrm{~m}$ de la Universidad Laboral de Tarragona, el Prof. Torroja, en

(*) La descripción de la estructura y los resultados de los ensayos pueden verse en "Die Schalenkuppel in vorgenspanntem Beton der Kirche Felix und Regula in Zürichn, por E. Schubiger. "Schweizerische Bauzeitung". 29 abril 1950.

(**) Los detalles de la estructura, así como las teorías utilizadas para dimensionarla, fueron presentados al último Congreso de Hormigón Pretensado, celebrado en Amsterdam, en un trabajo titulado: "Research on a prestressed concrete northlight shell", by Prof. Dr. Ir. A. M. Haas.

(***) Los resultados de los ensayos fueron publicados en el número 81 de "Informes de la Construcción», en un artículo titulado: "Ensayo en modelo reducido, de una cubierta laminar de hormigón pretensado», por C. Benito. Mayo 1956. 

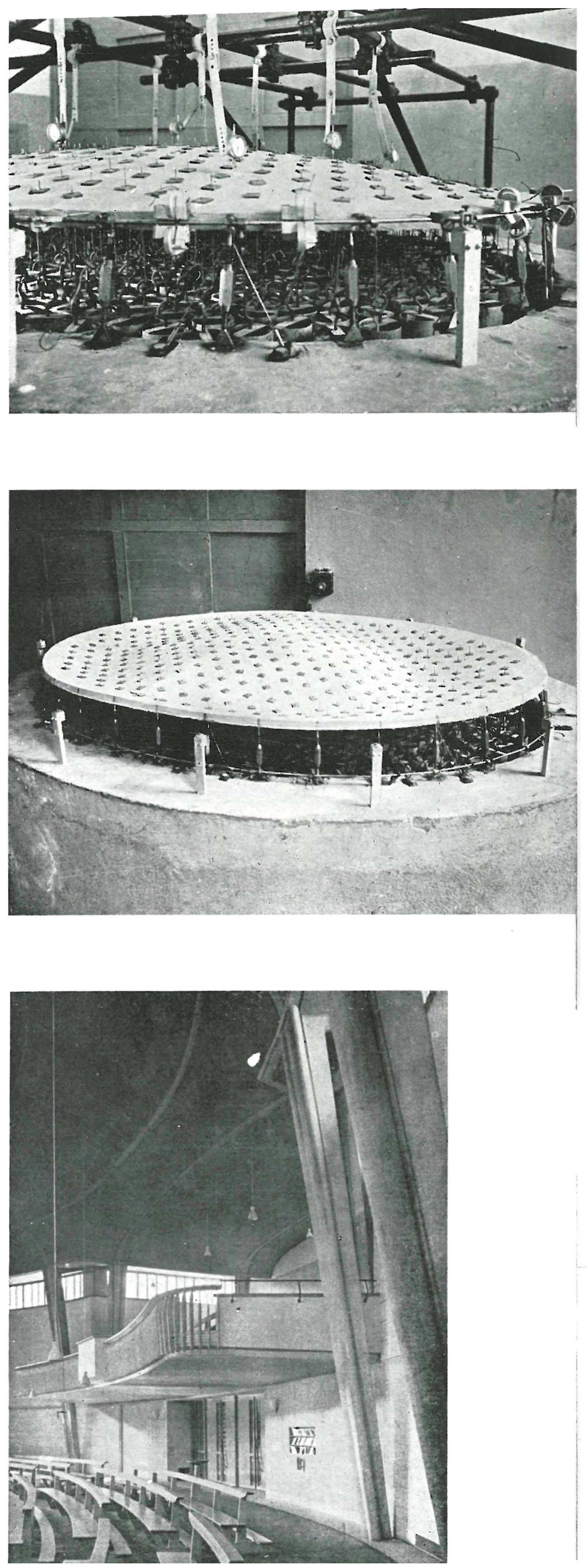

(C) Consejo Superior de Investigaciones Científicas Licencia Creative Commons 3.0 España (CC-by) 

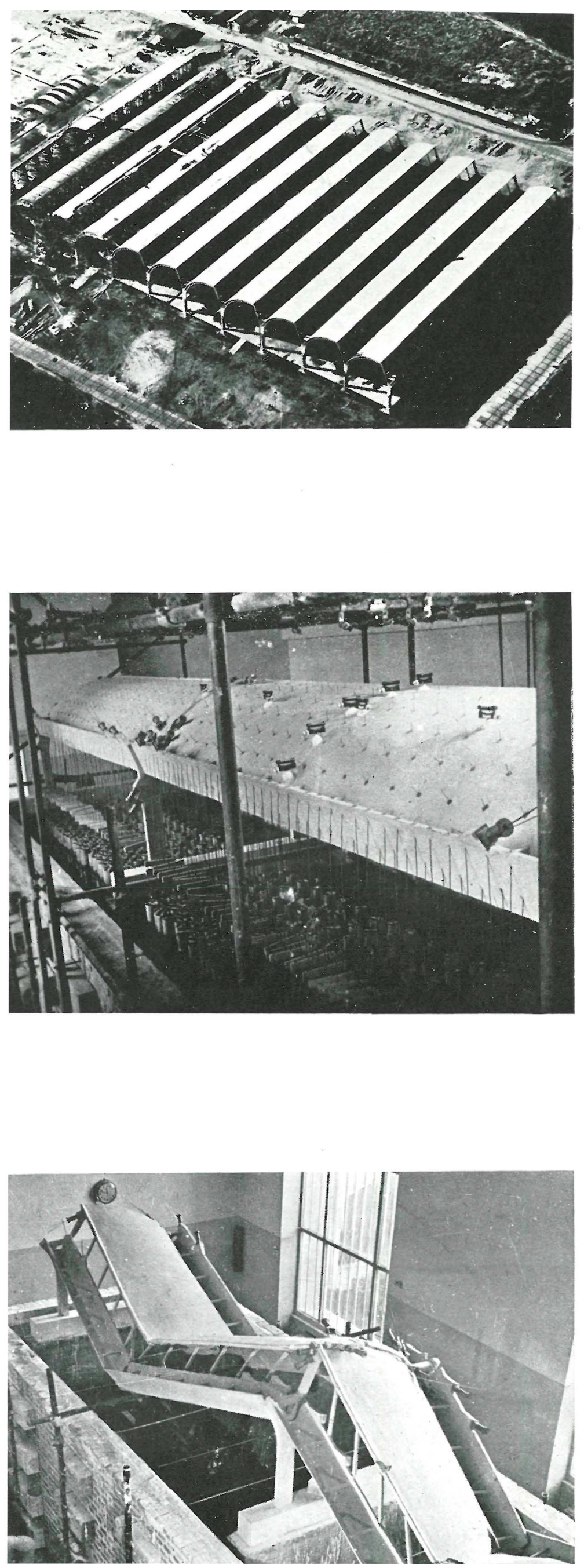

(C) Consejo Superior de Investigaciones Científicas Licencia Creative Commons 3.0 España (CC-by) 

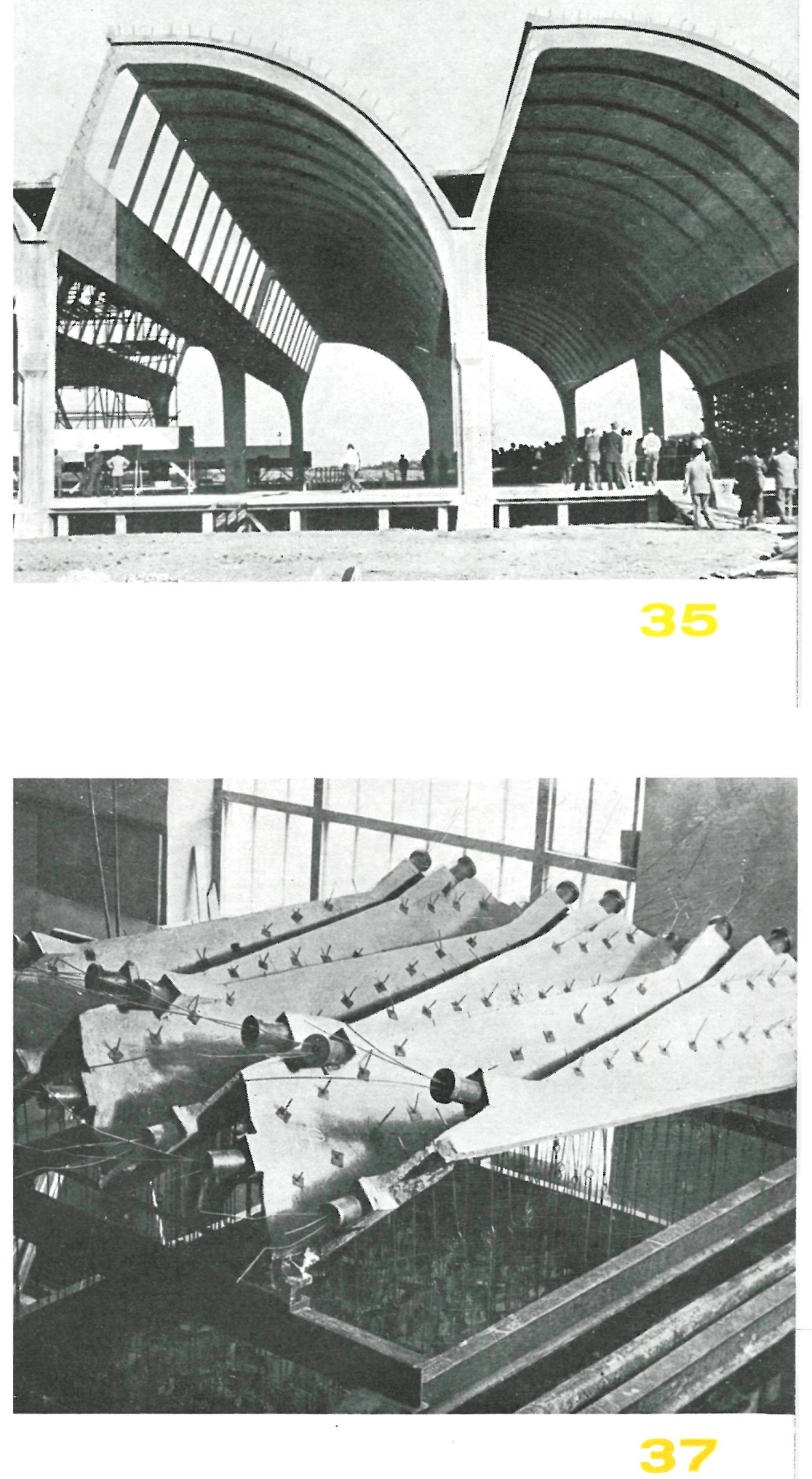

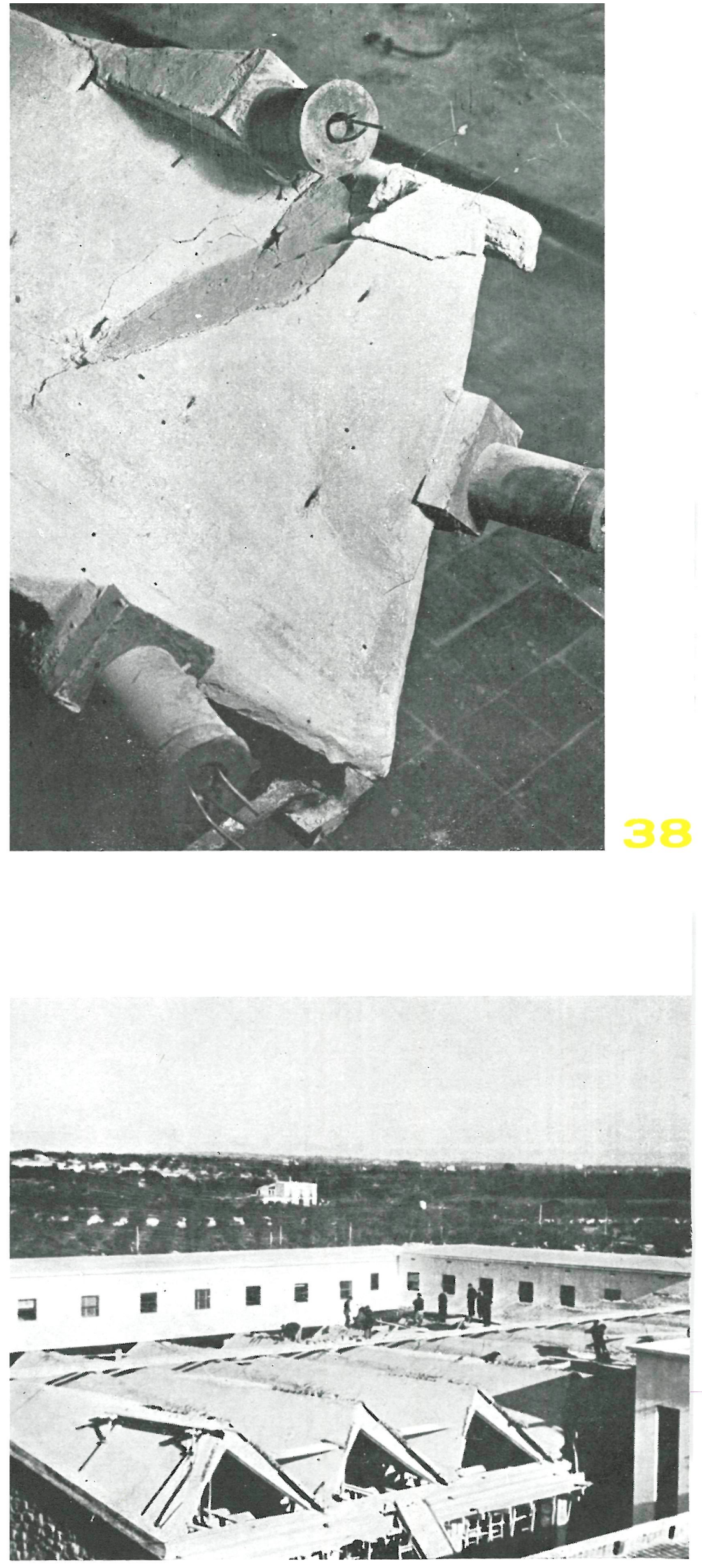


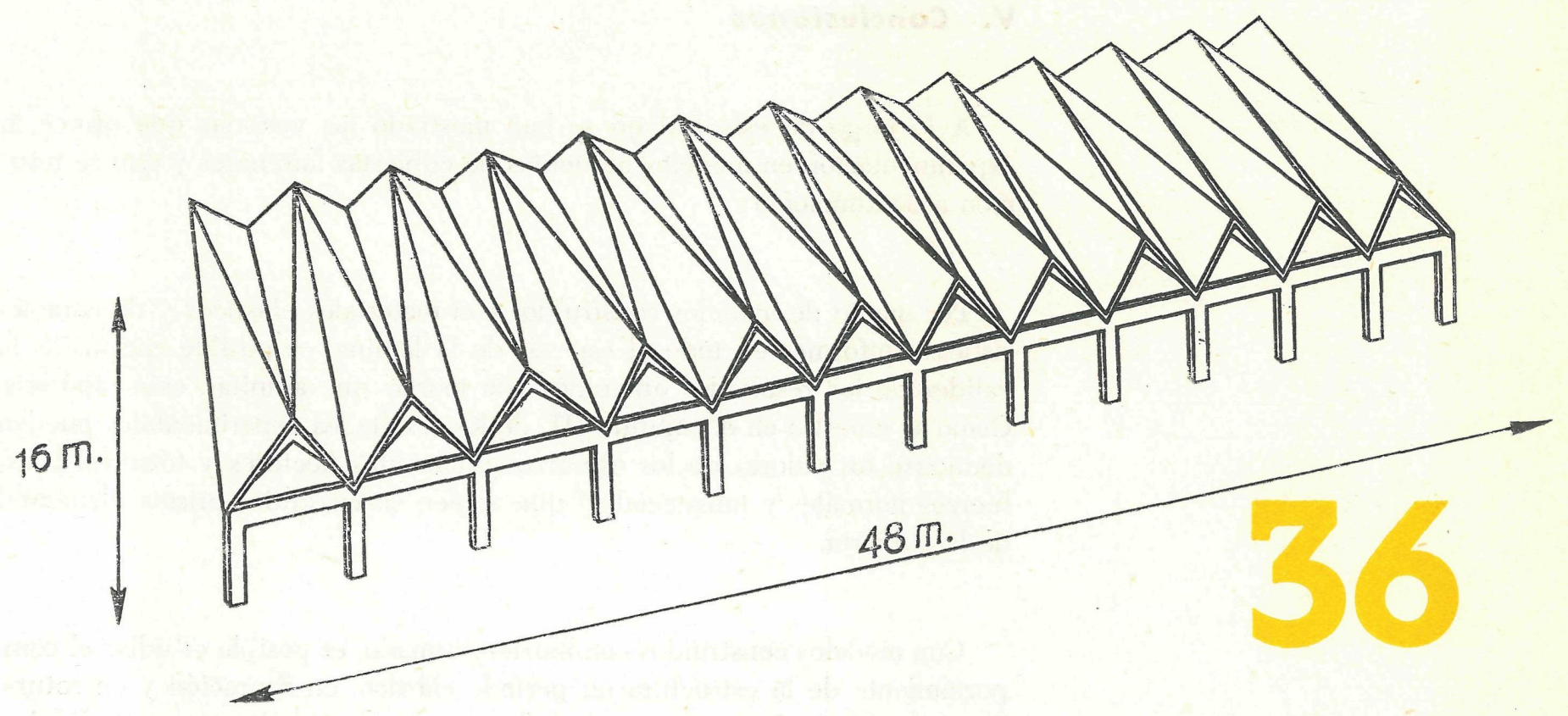

colaboración con los ingenieros F. del Pozo y A. Páez, desarrollaron el proyecto propuesto por el arquitecto A. de la Vega para una cubierta laminar plegada, de hormigón armado y postensado. Su forma puede verse en la figura 36.

La cubierta está formada por una serie de triángulos planos iguales, de $12 \mathrm{~cm}$ de espesor, que se empotran elásticamente entre sí, y se apoyan sobre pórticos triangulares, situados en las fachadas más largas del edificio, el cual queda totalmente libre de apoyos en su interior.

La repetición de elementos resistentes iguales nos permitió reproducir en el modelo un grupo de ellos, de tal forma que a su parte central no llegasen las influencias del borde libre de los últimos triángulos de cada extremo. El modelo (figura 37) se construyó a escala $1 / 15 \mathrm{y}$, como en los ejemplos anteriores, se reprodujeron en él las armaduras previstas por el autor del proyecto. Se le sometió a los efectos de cargas uniformes crecientes y decrecientes, se aumentó la carga hasta la fisuración y, por último, se incrementó hasta rotura. La figura 38 reproduce un aspecto parcial de la rotura, y en la 39 se ve la obra en un estado avanzado de construcción. Para el anclaje de los alambres de postensado, tanto en el modelo como en la obra real, se utilizaron anclajes Barredo.

Aunque podríamos incluir otros ejemplos, esperamos que lo expuesto sirva para mostrar las ventajas de la experimentación en modelos reducidos a rotura, cuando se trate de proyectar estructuras laminares. 


\section{v. Conclusiones}

A lo largo de este trabajo se han mostrado las ventajas que ofrece la experimentación en modelos reducidos de cubiertas laminares y que se resumen a continuación:

Por medio de modelos construídos con materiales elásticos y de características uniformes en todo el espesor de la lámina, es posible contrastar la validez de los resultados obtenidos con teorías que admitan esas hipótesis. Como se muestra en el capítulo III, de los resultados experimentales pueden deducirse los valores de los esfuerzos (momentos flectores y torsores, y esfuerzos normales y tangenciales) que actúen en cualquier prisma elemental de la cubierta.

Con modelos construídos con mortero armado, es posible estudiar el comportamiento de la estructura en período elástico, en fisuración y en rotura. También puede determinarse el coeficiente de seguridad a rotura de la obra proyectada. Buena prueba de ello son los ejemplos que se incluyen en el capítulo IV.

Por encima de estas ventajas, ya de por sí importantes, está la posibilidad de observar el comportamiento resistente de la estructura concebida, su modo de reaccionar bajo el efecto de las cargas. La compenetración del proyectista y su obra es, a nuestro juicio, el resultado más interesante de los ensayos en modelo reducido. De ellos, unas veces saldrán indicaciones para la reforma de determinadas zonas peligrosas, que tal vez estaban insuficientemente dimensionadas, y otras, la confirmación del acierto del proyectista. En todos los casos, el resultado es igualmente satisfactorio, pues proporciona la comprobación necesaria a toda construcción bien proyectada.

$\mathrm{El}$ autor desea hacer constar las satisfacciones que le ha proporcionado la colaboración del personal del Laboratorio Central, y muy especialmente de su Director y Maestro, don Eduardo Torroja; de los Ayudantes de Obras Públicas, don Antonio Sánchez Vegas y don Fernando Rodríguez Merodio, encargados de la construcción de los modelos del mortero, y del Perito Industrial, don Anselmo Moreno Castillo, encargado de la construcción de modelos elásticos y de la medida en todos los modelos realizados. A ellos corresponden los méritos principales de estos trabajos. 